\title{
BENCHMARKING STIFF ODE SOLVERS FOR ATMOSPHERIC CHEMISTRY PROBLEMS-I. IMPLICIT VS EXPLICIT
}

\author{
A. SANDU*, ${ }^{*}$ J. G. VERWER $\dagger$, M. VAN LOON $\ddagger$, G. R. CARMICHAEL $\S$, \\ F. A. POTRA $\uparrow$, D. DABDUB $\|$ and J. H. SEINFELD** \\ * Program in Applied Mathematics and Computational Sciences, The University of Iowa, Iowa City, \\ IA 52246, U.S.A.; † Center for Mathematics and Computer Science (CWI), P.O. Box 94079, 1090 GB \\ Amsterdam, The Netherlands; $\ddagger$ Center for Mathematics and Computer Science (CWI), P.O. Box 94079, \\ 1090 GB Amsterdam, The Netherlands; §Center for Global and Regional Environmental Research \\ and the Department of Chemical and Biochemical Engineering. The University of Iowa, Iowa City, \\ IA 52246, U.S.A.; I Departments of Mathematics and Computer Science. The University of lowa, \\ lowa City, IA 52246, U.S.A.; Department of Mechanical and Aerospace Engineering, University \\ of California Irvine, Irvine, CA 92717-3975, U.S.A.; ** Department of Chemical Engineering. \\ California Institute of Technology, Pasadena, CA 91125, U.S.A.
}

(First received 29 February 1992 and in final form 6 June 1996. Published July 1997)

\begin{abstract}
In many applications of atmospheric transport-chemistry problems, a major task is the numerical integration of the stiff systems of ordinary differential equations describing the chemical transformations. This paper presents a comprehensive numerical comparison between five dedicated explicit and four implicit solvers for a set of seven benchmark problems from actual applications. The implicit solvers use sparse matrix techniques to economize on the numerical linear algebra overhead. As a result they are often more efficient than the dedicated explicit ones, particularly when approximately two or more figures of accuracy are required. In most test cases, sparse RODAS, a Rosenbrock solver, came out as most competitive in the $1 \%$ error region. Of the dedicated explicit solvers, TwOSTEP came out as best. When less than $1 \%$ accuracy is aimed at, this solver performs very efficiently for tropospheric gas-phase problems. However, like all other dedicated explicit solvers, it cannot efficiently deal with gas-liquid phase chemistry. The results presented may constitute a guide for atmospheric modelers to select a suitable integrator based on the type and dimension of their chemical mechanism and on the desired level of accuracy. Furthermore, we would like to consider this paper an open invitation for other groups to add new representative test problems to those described here and to benchmark their numerical algorithms in our standard computational environment. (C) 1997 Elsevier Science Ltd.
\end{abstract}

Key word index: Atmospheric chemistry, air pollution modeling, numerical stiff ODEs, benchmarking, dedicated explicit solvers, sparse implicit solvers.

\section{INTRODUCTION}

To better understand the transport and fate of trace gases and pollutants in the atmosphere, comprehensive atmospheric transport-chemistry models have been developed. For their numerical solution, very often the operator splitting approach is followed. A major computational task is then the numerical integration of the stiff ODE (ordinary differential equation) systems describing the chemical transformations. These systems take the special production-loss form

$$
\begin{aligned}
\frac{\mathrm{d} y}{\mathrm{~d} t} & =f(t, y):=P(t, y)-L(t, y) y, \\
y(t) & =\left(y_{1}(t), \ldots, y_{m}(t)\right)^{\mathrm{T}},
\end{aligned}
$$

\footnotetext{
${ }^{1}$ Author to whom Correspondence should be addressed.
}

where $L(t, y)$ is a diagonal matrix. The integration must be carried out repeatedly at all spatial grid points for all split intervals chosen. Consequently, a full transport-chemistry computation involves a huge number of stiff ODE integrations. Although the numerical stiff ODE field is well developed and a variety of efficient and quite reliable stiff ODE solvers is available (Hairer and Wanner, 1991), the general experience is that still faster solvers are needed. There are many such solvers in use in atmospheric models. Often the numerical methods are adapted for a particular chemistry scheme, like QSSA methods (Hov et al., 1978; Hesstvedt et al., 1978; Verwer and Simpson, 1995). In such a case a change in the chemistry scheme necessitates a reconsideration of the numerical scheme as well, which is a disadvantage. Furthermore, this adaptation makes it very hard to really assess and compare the numerical efficiency and accuracy for different solvers. Continuing our previous 
work (see Jay et al., 1995; Verwer et al., 1996 and the references therein), the purpose of the current paper is a comprehensive benchmark comparison between a number of solvers which have been proposed in the literature. We apply them as normal ODE solvers without any adaptation for the chemistry scheme.

The paper is organized as follows. In Section 2 we briefly describe our test set. This set consists of seven problems based on three tropospheric gas-phase chemistry schemes, namely a small, a medium and a large scheme, one stratospheric scheme coming from NASA, and one hybrid gas-liquid phase scheme from cloud modeling which we obtained from Matthijsen (1995). Because of the diverse applications, these chemical schemes constitute a representative test set for evaluating and comparing numerical solvers. Section 3 is devoted to the solvers. We have tested nine existing solvers, namely TwOSTEP (Verwer, 1994; Verwer et al., 1996; Verwer and Simpson 1995), CHEMEQ (Young and Boris, 1977), the most simple QSSA solver, an extrapolated QSSA solver (Jay et al., 1995), the QSSA solver ET based on the extrapolation technique of Dabdub and Seinfeld (1995), as well as the implicit solvers LSODES (Hindmarsh, 1983), VODE (Brown et al., 1989), SDIRK4 (Hairer and Wanner, 1991) and RODAS* (Hairer and Wanner, 1991).

The first five of these are special purpose and compute the solution in an explicit way, as opposed to the last four which are all implicit and state-of-the-art in the numerical stiff ODE field. For our purpose, the solvers VODE, SDIRK 4 and RODAS have been provided with a sparse-matrix technique (Sandu et al., 1996) to economize on the numerical algebra overhead in the modified Newton solution. LSODES has already built in a sparse-matrix technique and is a successor of LSODE (Hindmarsh, 1983) which is popular amongst atmospheric chemists as a reference code. By providing the implicit solvers with sparse-matrix techniques, they belong to the fastest of their kind. In Section 4 we describe the setup of the experiments. Section 5 contains the results of the comparisons and the final Section 6 collects a number of general remarks and final conclusions.

All experiments discussed in this paper were carried out on a single-processor workstation and concern box-model tests. We emphasize that promising solvers should also be compared in actual $3 \mathrm{D}$ transport applications where the issues of memory use, vectorization (Jacobson and Turco, 1994; Verwer et al., 1996) and parallelization are of great practical importance. Code changes connected with vectorization and/or parallelization for a particular architecture can result in CPU time decreases of orders of magnitude.

* RODAS is a Runge-Kutta-Rosenbrock solver and hence not implicit in the strict mathematical sense. However, a Rosenbrock method requires the solution of linear system of algebraic equations, like an implicit method does if the iterative Newton technique is used. For ease of presentation, in this paper we therefore also call RODAS implicit.
To enable interested readers to further extend this benchmark comparison using their own solvers, as well as to extend our problem set with other challenging example problems from atmospheric chemistry, all the software we have used for the problems and the solvers has been put on a ftp-site (see ftp. cgrer.uiowa.edu. (1996) and the instructions therein).

\section{THE BENCHMARK PROBLEMS}

The seven test problems are based on a set of five chemical schemes which are presently being used in various studies. Four of them describe gas-phase, and one describes gas-liquid phase chemistry. All are fully documented elsewhere. Before briefly describing each problem, several general remarks are in order:

- All the test problems were uniformly coded in FORTRAN using the KPP symbolic preprocessor (Damian-Iordache and Sandu, 1995). This uniformity is important for a meaningful intercomparison, since part of the algorithms need the derivative function in production-destruction form, part need it in the standard form, and some of them need an analytical Jacobian. None of the solvers was favored/inhibited by a cheaper/more expensive implementation of these functions. FORTRAN code defining the test problems can be obtained from ftp.cgrer.uiowa.edu. (1996).

- All problems were run for five days. This time interval is sufficiently large for taking into account several diurnal cycles originating from the photochemical reactions. For all models the unit of components of $\mathrm{d} y / \mathrm{d} t$ used in the numerical tests is number of molecules $\mathrm{cm}^{-3} \mathrm{~s}^{-1}$.

- The tropospheric gas-phase problems are based on three different chemical schemes. These are the 15-species EUSMOG scheme, the 32-species CBM-IV scheme, and the 84-species mechanism implemented in the STEM-II model. This mechanism will be referred to as AL. All three are used in present applications and are representative of those being used in the atmospheric chemistry models. Varying the size of the mechanism is important since both implicit and explicit solvers are considered for this benchmark. The stratospheric gas-phase problem contains 34 species and the tropospheric gas-liquid phase one 65 .

- The same urban and rural scenarios are simulated with CBM-IV and with AL. Although the chemical conditions are identical and the calculated results very close, the performances of the numerical solvers depend on the chemical mechanism used. We will make this point later in the paper.

- An important issue in our numerical comparison is the use of a sparse matrix technique (Sandu et al., 1996) to economize on the linear algebra costs which the stiff solvers spend in the modified Newton iteration. As a measure of these costs, we give in Table 1 the number of nonzero elements in the Jacobian matrix, as well as the number of nonzero entries in the 
Table 1. The dimension of the test problems, the number of nonzeroes in the Jacobian matrix, and the number of nonzeroes in the Newton matrix after the LU factorization (difference between the numbers in the third and second row is the fill-in)

\begin{tabular}{lrrrrr}
\hline Problem & A & B, C & D, E & F & G \\
\hline Dimension & 15 & 32 & 84 & 34 & 65 \\
Jacobian & 57 & 276 & 674 & 246 & 506 \\
Factorized & 57 & 300 & 768 & 280 & 629 \\
\hline
\end{tabular}

Newton matrix after the LU factorization. The ratio between the number of nonzeroes in the Jacobian matrix and the square of the dimension gives an indication to which extent a sparse matrix solution may improve the timing compared to a standard dense matrix solution. If this ratio is small, say less than about $1 / 4$, and a reordering of the species exists which gives rise to a small fill-in after the factorization, then a good sparse solution technique will be significantly more efficient than the standard dense solution. The table shows that for our test problems both the ratio for the Jacobian and the resulting fill-in are quite small. The sparse matrix technique we have used is based on a diagonal Markowitz criterion (see Section 3.5 for some more details). Lest we miss the obvious, for problems of a small dimension for which the dense matrix numerical algebra costs are not dominating, the gain in using a more efficient matrix solution will be hardly noticeable in the overall costs. This is the case for Problem A (see also Verwer et al., 1996).

The dedicated explicit algorithms are scalarly implicit and exploit the production-loss form of the ODE system. They are based on the assumption that all short-lived species, causing the problem to be stiff, are only weakly coupled to all other species. In mathematical terms this implies that for these short-lived species the loss term $-L_{i}(t, y)$ is close to a stiff eigenvalue of the Jacobian matrix, and that no stiff eigenvalues exist which are not close to a loss term. Following McRae et al., (1982), the following reasoning explains this. Consider atomic oxygen $O$ which is a very fast reacting species. In many models a typical predominant removal step for $\mathbf{O}$ (by some orders of magnitude) is reaction with $\mathrm{O}_{2}$ and a third body $M$, i.e.

$$
\mathrm{O}+\mathrm{O}_{2}+\mathrm{M} \stackrel{k}{\longrightarrow} \mathrm{O}_{3}+\mathrm{M} \text {. }
$$

The kinetics for $O$ is then well described by the scalar ODE

$$
\frac{\mathrm{d}[\mathrm{O}]}{\mathrm{d} t}=P_{[\mathrm{O}]}-L_{[0]}[\mathrm{O}], \quad L_{[\mathrm{O}]}=k\left[\mathrm{O}_{2}\right][\mathrm{M}]
$$

where $L_{[0]}$ is a constant since the concentrations of $\mathrm{O}_{2}$ and $\mathrm{M}$ are fixed. Hence there exists no coupling with other species through the loss rate. Because for atomic oxygen coupling through the production rate
$P_{[0]}$ is very weak too, the predominance of the above reaction now trivially implies that $-L_{[0]}$ is close to a stiff eigenvalue of the Jacobian matrix of the whole system and, in addition, that in first approximation the exact solution for $O$ is given by

$[\mathrm{O}](t+h)=\frac{P_{[0]}}{L_{[0]}}+\left([\mathrm{O}](t)-\frac{P_{[\mathrm{O}]}}{L_{[0]}}\right) \mathrm{e}^{-L_{[0]^{h}}}$

This exact solution for truly constant coefficients forms the starting point for the popular QSSA methods.

Although this explanation is not mathematically rigorous for the nonlinear problems we deal with, it predicts to a great extent whether an explicit solver of the type used in this paper can be justified in advance*. For each problem we therefore illustrate the eigenvalue relationship in a table showing the species and eigenvalues for which the relationship is found to exist and the distribution of the remaining part of the spectrum $\dagger$ of the Jacobian (see also Fig. 8 in McRae et al. (1982)). In this remaining part, eigenvalues thus can be of two sorts, either they are small and hence do not introduce stiffness, or they are large but cannot be associated with a single short-lived species. If these latter eigenvalues exist, then the special purpose explicit methods can fail completely. Inspection of all the tables presented here will reveal that these latter eigenvalues exist only for the tropospheric wet problem $G$. This observation is in line with our test results.

\subsection{Problem A: The EUSMOG model}

This problem is borrowed from a model which is currently implemented and tested at the CWI in a Dutch smog prediction code in the framework of the project EUSMOG (van Loon, 1995a, b). The problem is a highly parameterized version of the EMEP MSC-W ozone chemistry scheme (Simpson et al., 1993). It consists of 15 reactions between 15 species and is extensively described in van Loon (1995a). It has been used before in the comparisons reported in Verwer et al. (1996), where it has also been documented. Information about the eigenvalues can be found in Table 2.

\subsection{Problems $B$ and $C$ : the CBM-VI model}

These problems are based on the Carbon Bond Mechanism IV (CBM-IV) (Gery et al., 1989), consisting of 32 chemical species involved in 70 thermal and 11 photolytic reactions. The concentration of $\mathrm{H}_{2} \mathrm{O}$ was held fixed throughout simulation. The CBM-IV mechanism was designed for the numerical simulation of

* For one of these, namely TwOSTEP using Gauss-Seidel iteration, the coupling between short- and long-lived species may be stronger, since Gauss-Seidel iteration introduces a form of "triangular implicitness".

$\dagger$ All the eigenvalues were computed with the routine dgees from LAPACK. 
Table 2. Distribution of real part of the spectrum of the Jacobian for EUSMOG problem A

\begin{tabular}{lc}
\hline Problem & A \\
\hline Species $i$ & $\operatorname{Re}\left(\dot{\lambda}_{i}\right)$ \\
OH & -9 \\
All other $\in$ & {$\left[-4 \times 10^{-4}, \approx 0\right]$} \\
\hline
\end{tabular}

chemical processes in urban and in regional-scale models. Test problem $B$ describes an urban scenario and simulates a heavily polluted atmosphere. The initial concentrations and the levels of hourly emissions follow those described in Saylor and Ford (1995). This helps to relate our results to those presented in the above-mentioned paper. Test problem $\mathrm{C}$ describes a rural scenario and simulates a clean atmosphere. It follows the IPCC Chemistry Intercomparison Study, third Bio scenario (see Prather et al. (1995)). The values of initial concentrations and the values of hourly emissions are given in Table 5. The emission was released in equal quantities at the beginning of each time interval. Information about the stiffness of the models in terms of the eigenvalues of the Jacobian is presented in Table 3.

\subsection{Problems $D$ and $E$ : the AL model}

The test problems $D$ and $E$ are based on the largest chemical system tested here. They employ the kinetic mechanism that is presently used in the STEM-II regional-scale/transport/chemistry/removal model (Carmichael et al., 1986), consisting of 84 chemical species* involved in 142 thermal and 36 photolytic reactions. The mechanism, based on the work of Atkinson et. al. (1989) and Lurmann et al. (1986) can be used to study the chemistry of both highly polluted (e.g., near urban centers) and remote (e.g., marine) environments. Problem $D$ is an urban scenario, while problem $E$ a rural one, based on IPCC scenario 3. The simulated conditions and initial concentrations are identical to those employed in problems $\mathrm{B}$ and $\mathrm{C}$, respectively. The values of initial concentrations, and the values of hourly emissions are given in Table 5. The emission was performed in equal quantities at the beginning of each time interval. Information about the stiffness of the models in terms of the eigenvalues of the Jacobian is given in Table 4. Since AL does not treat explicitly $O\left({ }^{1} D\right)$ and $O\left({ }^{3} P\right)$, the large negative eigenvalues associated with these species are not present.

\subsection{Problem F: a stratospheric model}

This test problem is based on the chemical mechanism that has been used in the NASA HSRP/AESA stratospheric models intercomparison. The initial

- Plus 4 species whose concentrations were held fixed throughout simulation: $\mathrm{H}_{2} \mathrm{O}, \mathrm{CO}_{2}, \mathrm{O}_{2}, \mathrm{H}_{2}$.
Table 3. Distribution of real part of the spectrum of the Jacobian for CBM-IV problems $B$ and $C$

\begin{tabular}{lcc}
\hline Problem & B (urban) & $C$ (rural) \\
\hline Species $i$ & $\operatorname{Re}\left(i_{i}\right)$ & $\operatorname{Re}\left(i_{i}\right)$ \\
$O\left({ }^{1} D\right)$ & $-8.11 \times 10^{8}$ & $-8.11 \times 10^{8}$ \\
$O\left({ }^{3} P\right)$ & $-8.26 \times 10^{4}$ & $-8.26 \times 10^{4}$ \\
ROR & $-2.47 \times 10^{3}$ & $-2.46 \times 10^{3}$ \\
OH & -46 & -3.5 \\
TO & -4.27 & -4.2 \\
All other $\in$ & {$[-1.5, \approx 0]$} & {$[-0.14 . \approx 0]$} \\
\hline
\end{tabular}

Table 4. Distribution of real part of the spectrum of the Jacobian for AL problems D and $E$

\begin{tabular}{lcc}
\hline Problem & $\mathrm{D}$ (urban) & E (rural) \\
\hline Species $i$ & $\operatorname{Re}\left(\dot{\lambda}_{i}\right)$ & $\operatorname{Re}\left(\lambda_{i}\right)$ \\
$\mathrm{OH}$ & -28 & -1.81 \\
$\mathrm{CRO}_{2}$ & -1.45 & -1.38 \\
$\mathrm{CHO}_{2}$ & -1.45 & -1.30 \\
$\mathrm{MAOO}$ & -1.23 & -1.17 \\
$\mathrm{MVKO}$ & -1.23 & -1.17 \\
MCRG & -1.23 & -1.17 \\
All other $\in$ & {$[-0.3 . \approx 0]$} & {$[-0.03, \approx 0]$}
\end{tabular}

concentrations and the values of the rate constants follow the NASA region A scenario* with the difference that the photolysis rates were piecewise linearly interpolated. There are 34 species $\dagger$ involved in 84 thermal and 25 photolytic reactions. The values of initial concentrations for the most important species are given in Table 6. No emissions have been prescribed. For a complete description of the problem we refer to the NASA ftp site. Information about the stiffness of the problem in terms of the eigenvalues of the Jacobian is given in Table 7.

\subsection{Problem G: a wet model}

From the numerical point of view, this test problem is the most difficult one. It contains 65 species $\ddagger$ involved in 77 thermal and 11 photolytic gas-phase chemical reactions, 39 liquid-phase chemical reactions and 39 gas-liquid mass transfer reactions. The gas-phase mechanism is based on CBM-IV, while the liquid-phase mechanism is based on a chemical scheme the authors obtained from Matthijssen (1995). Initial concentrations are given in Table 9. Information about the stiffness of the problem in terms of the eigenvalues of the Jacobian is given in

* Model available at NASA ftp site, contact Douglas E. Kinnison, kinnison1@llnl.gov.

†Plus 6 species whose concentrations were held fixed throughout simulation: $\mathrm{H}_{2} \mathrm{O}, \mathrm{CO}, \mathrm{O}_{2}, \mathrm{H}_{2}, \mathrm{~N}_{2}, \mathrm{CH}_{4}$

$\ddagger$ Plus 5 species whose concentrations were held fixed: $\mathrm{H}_{2} \mathrm{O}$ (vapour), $\mathrm{H}_{2} \mathrm{O}$ (drops), $\mathrm{CH}_{4}, \mathrm{O}_{2}, \mathrm{CO}_{2}$ (aq). 
elsewhere and will be discussed here only very briefly. The explicit solvers are not standard. For these we will therefore give the underlying integration formulas. All solvers use variable step size control. To save space, implementation details on the variable step size control are omitted. The interested reader is referred to ftp.cgrer.uiowa.edu. (1996), from where for each solver and each driver program used our FORTRAN code can be obtained. Before briefly describing the solvers, several general remarks are in order:

- The explicit solvers are applied to the given ODE system (1) without problem-dependent modifications. Modifications, such as lumping, can improve their performance notably (see e.g. Verwer et al., 1996 where this is illustrated for the EUSMOG problem), but have the disadvantage of being problem dependent. With lumping we mean here the technique of grouping species into chemical families to reduce, for example, the stiffness, or to enforce conservation for a chemical family (see Hov (1978) Hesstvedt et al. (1978) where this form of lumping was proposed first for the QSSA method). Hence our comparison emphasizes the numerical properties and performance of the solvers applied.

- The variable step size control requires the choice of a relative error tolerance rtol and an absolute error tolerance atol. The choices made for atol and rtol differ per solver and are not specified here. Noteworthy is that at certain times the concentrations of some species (e.g. radicals) can become smaller than $1.0 \mathrm{mlc} \mathrm{cm}{ }^{-3}$. Because these values are insignificant, they are ignored in the step size control.

- Often we also prescribe a minimal step size. The use of a minimal step size improves efficiency since extremely small steps can be selected by a variable step size selection scheme. Atmospheric chemistry problems containing photochemical reactions can possess time constants as small as $10^{-8}$ to $10^{-9}$ seconds and step size selection mechanisms do signal these. However, these extremely small step sizes are redundant because the minimal time constants of importance for photochemical chemistry models are of the order of seconds and species with a time constant truly smaller almost instantaneously get in their (solution dependent) steady state when they are perturbed. On the other hand, too large lower bounds for the step size can cause convergence and loss of accuracy problems to the numerical solvers.

- All sparse implicit solvers work with the analytical Jacobian matrix and can be shown to mimic conservation rules which exist for the $O D E$ system. This does not hold for all of the explicit solvers.

- A numerical comparison should focus on modest accuracies, say relative accuracies near $1 \%$. Higher accuracy levels are redundant for the actual practice of air pollution modelling.

\subsection{QSSA}

Two QSSA solvers were used. The first is based on the most simple QSSA formula

$$
\begin{aligned}
y^{n+1}= & e^{-h L\left(t_{n}, y^{n}\right)} y^{n} \\
& +\left(I-e^{-h L\left(l_{n}, y^{n}\right)}\right) L^{-1}\left(t_{n}, y^{n}\right) P\left(t_{n}, y^{n}\right),
\end{aligned}
$$

where $y^{n}$ denotes the numerical approximation at time $t=t_{n}$ and $h$ denotes the step size. This formula is explicit because the loss matrix $L$ is diagonal. The notion explicit thus means that no systems of algebraic equations need to be solved. The second is based on a Richardson extrapolated form of equation (2) and reads (Jay et al., 1995)

$$
\begin{gathered}
y_{h}^{n+1}=Q\left(y^{n} ; h\right), \quad y_{h / 2}^{n+1 / 2}=Q\left(y^{n} ; h / 2\right) \\
y_{h / 2}^{n+1}=Q\left(y_{h / 2}^{n+1 / 2} ; h / 2\right), \quad y^{n+1}=2 y_{h / 2}^{n+1}-y_{h}^{n+1}
\end{gathered}
$$

where $Q\left(y_{n} ; h\right)$ refers to equation (2). The extrapolated scheme is slightly more than two times expensive per time step of length $h$. The order of accuracy of equation (3) equals two, whereas equation (2) is of order one. See ftp.cgrer.uiowa.edu. (1996) for more details.

\subsection{CHEMEQ}

One of the first dedicated, explicit methods for solving chemical equations in comprehensive advection-reaction models is the hybrid algorithm of Young and Boris (1997). It is currently implemented in the CALGRID mesoscale model (Yamartino et al., 1992). In the original algorithm, the species are dynamically separated into two categories (fast and slow) according to the relative magnitude of their life-times $\tau_{k}=1 / L_{k}$ with respect to the current step size $h$. Each category is integrated with a special predictor-corrector formula. Our implementation of CHEMEQ follows the one described in Saylor and Ford (1995) and is based on the following predictor-corrector pairs (the abbreviation $P_{k}^{n}$ stands for $P_{k}\left(t_{n}, y^{n}\right)$, etc.):

- If $\tau_{k}>5 h$ (slow species):

$$
\begin{aligned}
\text { predictor: } y_{k}^{n+1}= & y_{k}^{n}+h\left(P_{k}^{n}-L_{k}^{n} y_{k}^{n}\right) \\
\text { corrector: } y_{k}^{n+1}= & y_{k}^{n}+\frac{n}{2}\left(P_{k}^{n}-L_{k}^{n} y_{k}^{n}\right. \\
& \left.+P_{k}^{n+1}-L_{k}^{n+1} y_{k}^{n+1}\right)
\end{aligned}
$$

- If $0.2 h \leqslant \tau_{k} \leqslant 5 h$ (intermediate species):

$$
\text { predictor: } y_{k}^{n+1}=\frac{y_{k}^{n}\left(2 \tau_{k}^{n}-h\right)+2 h P_{k}^{n} \tau_{k}^{n}}{2 \tau_{k}^{n}+h}
$$

corrector: $y_{k}^{n+1}$

$$
=\frac{y_{k}^{n}\left(\tau_{k}^{n}+\tau_{k}^{n+1}-h\right)+\frac{h}{2}\left(P_{k}^{n}+P_{k}^{n+1}\right)\left(\tau_{k}^{n}+\tau_{k}^{n+1}\right)}{\tau_{k}^{n}+\tau_{k}^{n+1}+h}
$$

- If $\tau_{k}<0.2 h$ (fast species):

$$
\text { steady-state assumption: } y_{k}^{n+1}=P_{k}^{n} / L_{k}^{n}
$$

A quick inspection will reveal that the corrector formulas are all derived from the implicit trapezoidal rule. They are applied, however, in an explicit manner. 
Hence, denoting $y_{k}^{(i)}$ as the $k$ th component of the $i$ th corrected approximation $y^{(i)}$ for $y^{n+1}$, in all occurrences $y_{k}^{(i)}$ is simply substituted in the right-hand sides of the corrector formulas, so as to compute the new approximations $y_{k}^{(i+1)}$. The correctors are applied until

$$
\max _{k}\left|\frac{y_{k}^{(i+1)}-y_{k}^{(i)}}{y y^{(i+1)}}\right| \leqslant 0.3 \text { tol, }
$$

where tol is an imposed tolerance value for the step size control which is based on atol and rtol. In case of non-convergence, the step is rejected and the computations restarted with $h_{\text {new }}=0.6 h$. In case of convergence the integration proceeds with $h_{\text {new }}=$ $h \min (1.1, \mathrm{fac})$, where fac $=\sqrt{\text { tol/err }}$ with err the estimation for the local truncation error. It is emphasized that the step selection in the original CHEMEQ is based uniquely on the convergence of corrector iterates, whereas we use an estimation for the local error to govern the step size. However, following CHEMEQ philosophy, a local error greater than tol does not force a step rejection. It only restricts $h_{\text {new }}$. See ftp.cgrer.uiowa.edu. (1996) for more details.

\section{3. $E T$}

The solver ET uses an extrapolation algorithm proposed in Dabdub and Seinfeld (1995). The approximations used for the extrapolation are computed with a predictor-corrector pair of which the corrector is a QSSA type formula. To describe the formulas used, we adopt the implicit notation used for CHEMEQ. Hence the evaluations of the right-hand side of the implicit formulas have to be thought of as carried out in the same way as for CHEMEQ. The predictor formula implemented in the solver tested in this paper is the simple QSSA formula

$$
y^{n+1}=e^{-h L^{n}} y^{n}+\left(I-e^{-h L^{n}}\right)\left(L^{n}\right)^{-1} P^{n} .
$$

Like for CHEMEQ, the corrector dynamically separates the species into three categories:

- If $\tau_{k}>100 h$, the trapezoidal rule is used,

$y_{k}^{n+1}=y_{k}^{n}+\frac{h}{2}\left(P_{k}^{n}-L_{k}^{n} y_{k}^{n}+P_{k}^{n+1}-L_{k}^{n+1} y_{k}^{n+1}\right)$.

If $0.1 h \leqslant \tau_{k} \leqslant 100 h$, the equations are corrected using the QSSA type formula

$$
y_{k}^{n+1}=\psi_{k}^{n+1}+\left(y_{k}^{n}-\psi_{k}^{n+1}\right) \exp \left[-\left(\frac{1}{L_{k}^{n}}+\frac{1}{L_{k}^{n+1}}\right) \frac{h}{2}\right] \text {, }
$$

where $\psi_{k}^{n+1}$ is defined by

$$
\psi_{k}^{n+1}=\frac{1}{4}\left(P_{k}^{n+1}+P_{k}^{n}\right)\left(\frac{1}{L_{k}^{n+1}}+\frac{1}{L_{k}^{n}}\right) .
$$
i.e.,

- If $\tau_{k}<0.1 h$, the steady-state assumption is made,

$$
y_{k}^{n+1}=\psi_{k}^{n+1} \text {. }
$$

For details about how the extrapolation is organized and the corrector is used we refer to Dabdub and Seinfeld (1995). See also ftp.cgrer.uiowa.edu. (1996) for more details.

\subsection{TWOSTEP}

TWOSTEP(Verwer, 1994; Verwer et al., 1996; Verwer and Simpson, 1995) is based on the variable step size, two-step backward differentiation formula (BDF)

$$
y^{n+1}=Y^{n}+\gamma h f\left(t_{n+1}, y^{n+1}\right), h=t_{n+1}-t_{n}, \quad \text { (12) }
$$

where $\gamma=(c+1) /(c+2), c=\left(t_{n}-t_{n-1}\right) /\left(t_{n+1}-t_{n}\right)$ and

$$
Y^{n}=\left((c+1)^{2} y^{n}-y^{n-1}\right) /\left(c^{2}+2 c\right) .
$$

The 2nd-order BDF formula (also denoted by BDF2) has been chosen in view of the modest accuracy requirement. Rather than using the common modified Newton iteration, the classical Gauss-Seidel or Jacobi iteration technique is used for computing an approximation to the implicitly defined $y^{n+1}$. In the application of these techniques we exploit, to some extent, the production-loss form equation (1), by which equation (12) can be written as

$$
\begin{aligned}
y^{n+1}= & F\left(y^{n+1}\right):=\left(I+\gamma h L\left(t_{n+1}, y^{n+1}\right)\right)^{-1} \\
& \times\left(Y^{n}+\gamma h P\left(t_{n+1}, y^{n+1}\right)\right) .
\end{aligned}
$$

The Gauss-Seidel technique is now applied to the nonlinear system of equations $y=F(y)$. That is, given the iterate $y^{(i)}$ as the $i$ th approximation for the sought solution $y^{n+1}$, TWOSTEP computes the next iterate $y^{(i+1)}$ by the componentwise formula

$y_{k}^{(i+1)}=F_{k}\left(y_{1}^{(i+1)}, \ldots, y_{k-1}^{(i+1)}, y_{k}^{(i)}, \ldots, y_{m}^{(i)}\right), k=1, \ldots, m$.

This results in an explicit computation owing to the diagonal form of $L$. More precisely, for the computation of $y_{k}^{(i+1)}$ only division by the scalar variable $1+\gamma h L_{k}\left(t_{n+1}, v\right)$ is required, where $v$ denotes the intermediate vector

$$
v=\left[y_{1}^{(i+1)}, \ldots, y_{k-1}^{(i+1)}, y_{k}^{(i)}, \ldots, y_{m}^{(i)}\right]^{\mathrm{T}} .
$$

The fact that in $v$ the first $(k-1)$ components are taken from the new iterate, makes equation (14) a Gauss-Seidel type iteration process. When we take all $m$ components in $v$ from the old iterate $y^{(i)}$, then an iteration method of Jacobi or Picard type results. Computationally, there is not much difference between the two, although Gauss-Seidel has to be programmed in line. Hence Jacobi iteration is somewhat easier to use. Here we will use both types of iteration techniques. Note that for the Gauss-Seidel technique the order of the species plays a role when only a small number of iterations are used. In all experiments we in fact restrict the number of iterations to only two. In Verwer et al. (1996) and Verwer and Simpson (1995) this has been shown to work well. 
TWOSTEP is based on a two-step formula which cannot be applied at an operator-split restart. At restart the one-step backward Euler formula is therefore used, which simply means that $Y^{n}=y^{n}$. The explicit iteration process is the same, so that there is no additional penalty at restart associated with extra linear algebra computations as is the case for implicit solvers using a Newton type iteration. TWOSTEP allows a maximal growth in step size at restart by a factor of two. This is less than onestep solvers usually allow, but still quite acceptable in view of the explicit iteration process. For more details on the code we refer to Verwer et al. (1996), Verwer and Simpson (1995) and ftp.cgrer.uiowa.edu. (1996).

\section{5. $V O D E$}

VODE is a "Variable coefficient Ordinary Differential Equation" solver based on the implicit BDF formulas (Brown et al., 1989; Hairer and Wanner, 1991) and a successor of the "Livermore Solver" LSODE from Hindmarsh (1983). The latter is popular in the field of atmospheric chemistry. For a discussion of the mathematical techniques implemented we refer to Brown et al. (1989), Hairer and Wanner (1991). We used VODE as a black box with its user parameter istart $=1$, except that we modified the code to carefully exploit the sparsity of the Jacobian matrix. This reduces the costs of solving the linear algebraic systems arising in the modified Newton iteration. In Jacobson and Turco (1994) and Verwer et al. (1996) it has been shown that this is very profitable for atmospheric chemistry problems. We used the sparse linear algebra implementation described in Sandu et al. (1996). The necessary routines are automatically generated by the symbolic chemical preprocessor KPP Damian-lordache and Sandu, 1995, which transparently:

- determines the sparse analytical Jacobian,

- reorders the species using a diagonal Markowitz criterion, in order to minimize the fill-in resulting from the LU decomposition of the matrix used in the modified Newton process,

- analyses the pattern of zeros in the Jacobian and builds the data structures needed for the sparse Doolittle LU decomposition,

- generates loop-free code for the forward-backward substitution routines.

The performance of VODE appeared to be sensitive to the choice of the absolute tolerances. Using the natural value atol $=1.0$ was not always optimal. We therefore set them componentwise as

$$
\text { atol }_{i}=\max \left(10^{-2}, 10^{-2} \mathrm{rtol} \eta_{i}\right) \quad\left(\mathrm{mlc} \mathrm{cm}^{-3}\right)
$$

where $\eta_{i}$ estimates the magnitude of the concentration of species $i$. See ftp.cgrer.uiowa.edu. (1996) for other specific parameter settings.

\section{6. $S D I R K 4$}

This solver has been borrowed from Hairer and Wanner (1991) where a full description along with numerical results can be found. It is based on a $4 \mathrm{th}$ order, diagonally implicit Runge-Kutta method using five stages. Because this solver is of one-step type, it allows a fast increase in step size after a restart. For atmospheric chemistry applications this is an obvious advantage. We have only modified it for the treatment of sparsity as described in Section 3.5. Hence all strategies were unaltered. See ftp.cgrer.uiowa.edu. (1996) for specific parameter settings.

\section{7. $R O D A S$}

This solver has also been borrowed from Hairer and Wanner (1991). It is based on a 4th order, Runge-Kutta-Rosenbrock method using six stages. This solver is also of one-step type and hence shares the advantage of a fast increase in step size after a restart with SDIRK 4 . The code has been modified for the treatment of sparsity as described in Section 3.5. All strategies were unaltered. See ftp.cgrer.uiowa.edu. (1996) for specific parameter settings.

\subsection{LSODES}

LSODES is a version of the popular BDF code LSODE which exploits the sparsity in the Jacobian matrix by calling linear algebra routines from the Yale Sparse Matrix Package (Eisenstat et al., 1977, 1982). It is obvious that VODE and LSODES are closely related. For our application an important difference is that VODE uses a dedicated sparsity technique, whereas LSODES uses the general Yale package, which is less efficient, in general. LSODE and LSODES are often used to solve atmospheric chemical kinetics equations (see e.g. Saylor and Ford, 1995). The code was applied with its user parameter setting $M F=121$, i.e. analytical Jacobian with an inner estimation of the sparsity structure. See ftp.cgrer.uiowa.edu. (1996) for other specific parameter settings.

\section{SETUP OF EXPERIMENTS}

The solvers are tested as if they were used in an operator splitting environment. This means that we split the total integration interval into $N$ subintervals of length $\Delta t$. For each subinterval we then restart the integration of the stiff solvers. For all test problems the length of the subintervals equals $\Delta t=3600 \mathrm{~s}$ leading to $N=120$ new starts over the $5 \mathrm{~d}$ period. The $3600 \mathrm{~s}$ subinterval means that we reckon with a $1800 \mathrm{~s}$ transport time step, assuming a Strang splitting symmetrized around the chemistry step. It should be stressed that the choice of the subinterval length is important for a box-model comparison, since this length determines the number of restarts. Restarts are expensive for implicit solvers using a Newton type iteration due to the linear algebra overhead. This 
holds in particular for the multistep solvers VODE and LSODES. For the one-step implicit solvers RODAS and SDIRK4 the penalty is less, since they are able to enlarge the step size after restart considerably faster. Generally, for any explicit solver the penalty is also less because of the absence of linear algebra overhead.

All test problems contain photochemical reactions. This means that part of the reaction constants are determined by time of the day dependent photolysis rates which undergo a rapid change at sunrise and sunset. This change gives rise to large variations in concentration values and normally force a solver to adjust the step size. In Problem $A$ the photolysis rates are given by a $C^{0}$ function, while in Problems B, C, D, $\mathrm{E}, \mathrm{F}$ and $\mathrm{G}$ by a $C^{1}$ function which are zero at nightly periods.

All the runs were made in double precision on a HP-UX 935 A workstation with a CPU clock frequency of $125 \mathrm{MHz}$ and $160 \mathrm{Mbytes}$ RAM. The numerical results for all test problems are compared with a very accurate reference solution computed by the code RADAU5 from Hairer and Wanner (1991) with the very tight tolerances $r$ tol $=10^{-12}$, atol ${ }_{i}=10^{-15} \eta_{i}$, where $\eta_{i}$ estimates the magnitude of the concentration of species $i$ in unit $\mathrm{mlc} \mathrm{cm}^{-3}$. Our measure of accuracy is based on a modified root mean square norm of the relative error. With the reference solution $y$ and the numerical solution $\hat{y}$ available at $\left\{t_{n}=t_{0}+n \Delta t, 0 \leqslant n \leqslant N\right\}$, we first compute for each species $k$

$$
\begin{gathered}
\qquad \mathrm{ER}_{k}=\sqrt{\frac{1}{\left|\mathscr{T}_{k}\right|} \sum_{n \in \mathscr{G}_{4}}\left|\frac{y_{k}\left(t_{n}\right)-\hat{y}_{k}\left(t_{n}\right)}{y_{k}\left(t_{n}\right)}\right|^{2}}, \\
\text { where } \mathscr{T}_{k}=\left\{0 \leqslant n \leqslant N:\left|y_{k}\left(t_{n}\right)\right| \geqslant a\right\} .
\end{gathered}
$$

Hence we compute specieswise a temporal error measure, which we then represent in the plots in two ways. Through the number of significant digits for the average of $E_{k}$, defined by

$$
\mathrm{SDA}_{1}=-\log _{10}\left(\frac{1}{m} \sum_{k=1}^{m} \mathrm{ER}_{k}\right)
$$

and the number of significant digits for the maximum of $E R_{k}$, defined by

$$
\operatorname{SDA}_{x^{\prime}}=-\log _{10}\left(\max _{k} \mathrm{ER}_{k}\right)
$$

The threshold factor $a$ used here is given the value $a=1.0 \mathrm{mlccm}^{-3}$. If the set $\mathscr{T}_{k}$ is empty, the value of $E R_{k}$ is neglected. The purpose of considering the above defined error measure instead of the root-mean square norm $(a=0)$ is to avoid chemically meaningless large relative errors for concentration values smaller than $1.0 \mathrm{mlc} \mathrm{cm}^{-3}$. It is instructive to present the accuracy of the computed results using averaged and maximal errors taken over the number of species. Finally, in the work-precision diagrams of the following section, efficiency is measured by CPU time. This is of course dependent on the computer architecture used. However, the relative magnitudes shown here should be applicable to scalar computers in general. We thus plot the SDA values against the measured CPU times on a logarithmic scale in unit seconds. Observe that $\mathrm{SDA}=2$ means $1 \%$ accuracy in the error measure used. In discussing the results presented in the next section we focus on this accuracy level. The different data points in the plots for the same solvers are associated to runs carried out for different relative tolerances.

\section{RESULTS AND ILLUSTRATIONS}

\subsection{Problem A: the EUSMOG model}

The work-precision diagram is given in Fig. 1. The implicit integrators and the two versions of TWOSTEP perform very well. For an accuracy of two digits, sparse VODE appears to be the fastest. The good performance of implicit integrators is due in part to the small dimension of the system which means less work with the linear algebra. Note that the JACOBI and (GAUSS-) SEIDEL versions of TWOSTEP have similar performance for this test problem (see also Verwer et al. (1996)). It is clear that the simplest QSSA solver, ET and CHEMEQ are the slowest among the tested routines. It is also worth noting that the QSSA performance greatly improves by extrapolation.

\subsection{Problems $B$ and $C$ : the CBM-IV model}

In Fig. 2 the numerical results for test problems $B$ and $C$ are presented. For obtaining two accurate digits, RODAS appears to be the fastest, followed by SDIRK, VODE, LSODES and TWOSTEP SEIDEL. The latter is the best when less accuracy is demanded, while the implicit codes are preferable for higher precisions. This is due to the fact that they use higher-order formulas (nicely represented by the higher slopes of their diagrams). An interesting remark is that the slope of the TWOSTEP JACOBI diagram decreases when higher accuracies are required. This is due to an imposed minimal step size of $0.01 \mathrm{~s}$, which makes the convergence of the Jacobi iteration very slow on part of the time interval. Since TWOSTEP was used with a fixed number of only two iterations, this is directly reflected in the accuracy of the solution. A minimal step size larger than $0.1 \mathrm{~s}$ creates similar problems in TWOSTEP SEIDEL. The gap between the two TWOSTEP diagrams is due primarily to the different values of the minimal step size used: $0.01 \mathrm{~s}$ for JACOBI and $0.1 \mathrm{~s}$ for SEIDEL. The explicit solver ET is not competitive at all. Its work-precision diagram is situated to the far right of Fig. 2. Among the other integrators, QSSA is clearly the slowest, but by extrapolation it gains about one accurate digit for the same CPU time. In both scenarios CHEMEQ performs better than the plain QSSA scheme but worse than Extrapolated QSSA. As expected, the LSODES and sparse VODE diagrams are similar, except that the LSODES one is shifted to the 


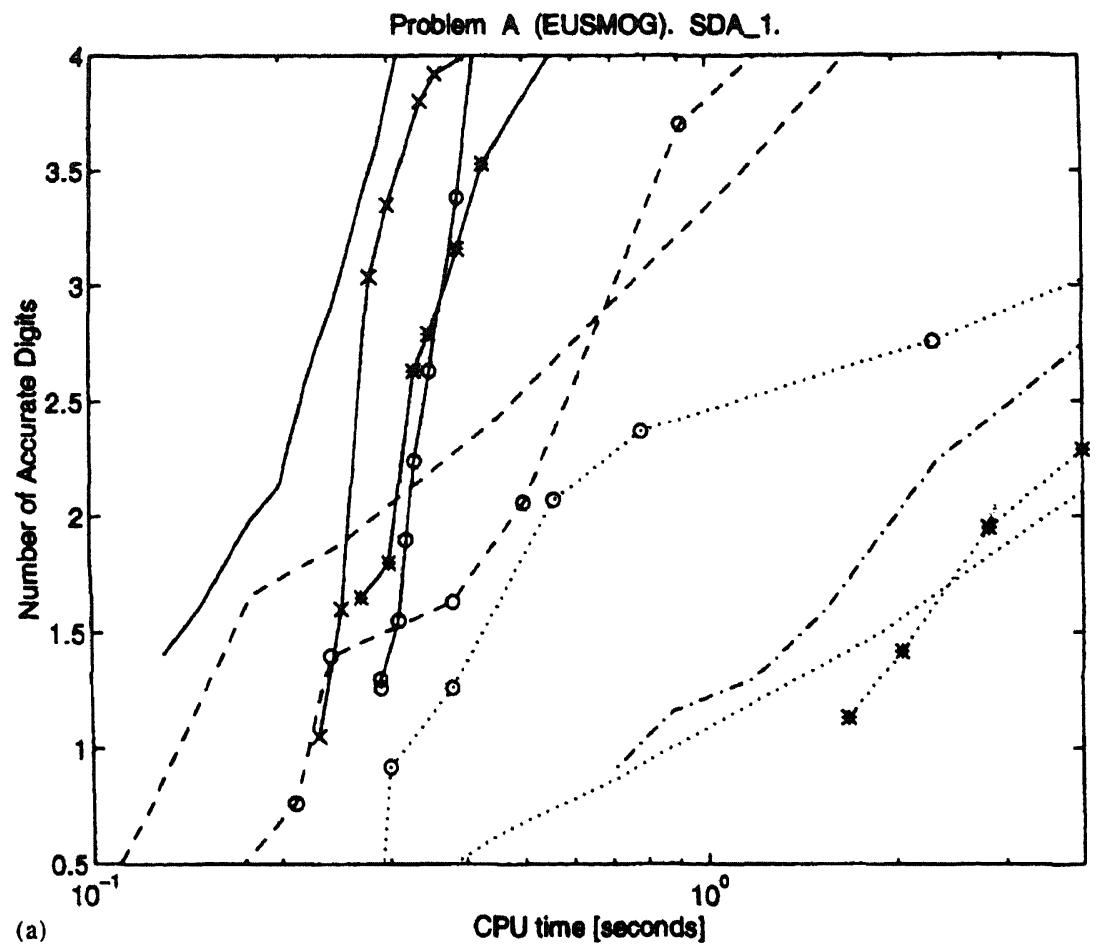

(a)

CPU time [seconds]

Problem A (EUSMOG). SDAinf.

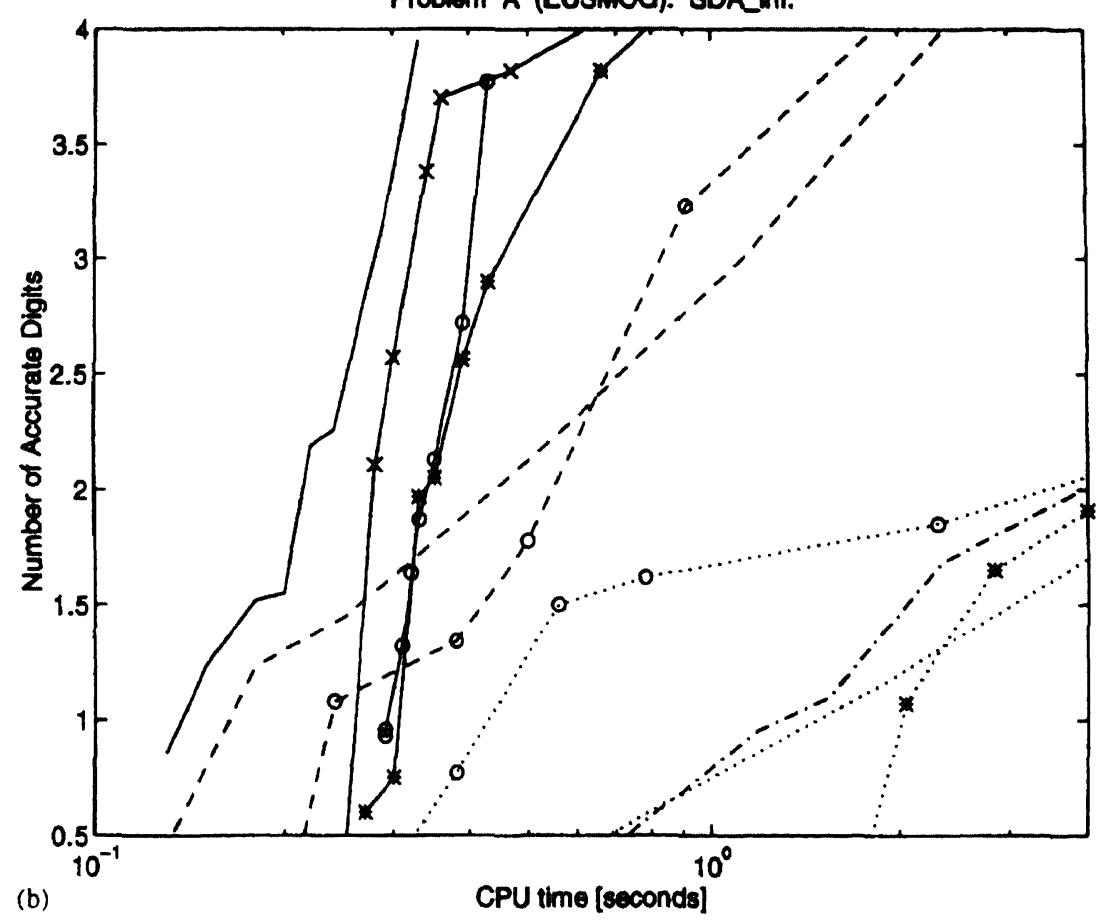

Fig. 1. Work-precision diagram for test problem A (EUSMOG) : TWOSTEP SEIDEL (dashed), TWOSTEP JACOBI (dashed with "O"), QSSA (dots), Extrapolated QSSA (dots with "O"), ET (dots with “*"), CHEMEQ (dash-dots), Sparse VODE (solid), Sparse SDIRK4 (solid with "*"), Sparse RODAS (solid with " $\times$ ") and LSODES (solid with "O").

right. Working with predefined sparsity data structures, VODE is consistently faster than the general purpose LSODES. However, despite its generality, for test problems $B$ and C LSODES performs very well.

\subsection{Problems $D$ and E: The AL model}

The results are reported in Fig. 3. It is interesting to compare code performances to those obtained for test problems $B$ and $C$, since the same urban and rural 

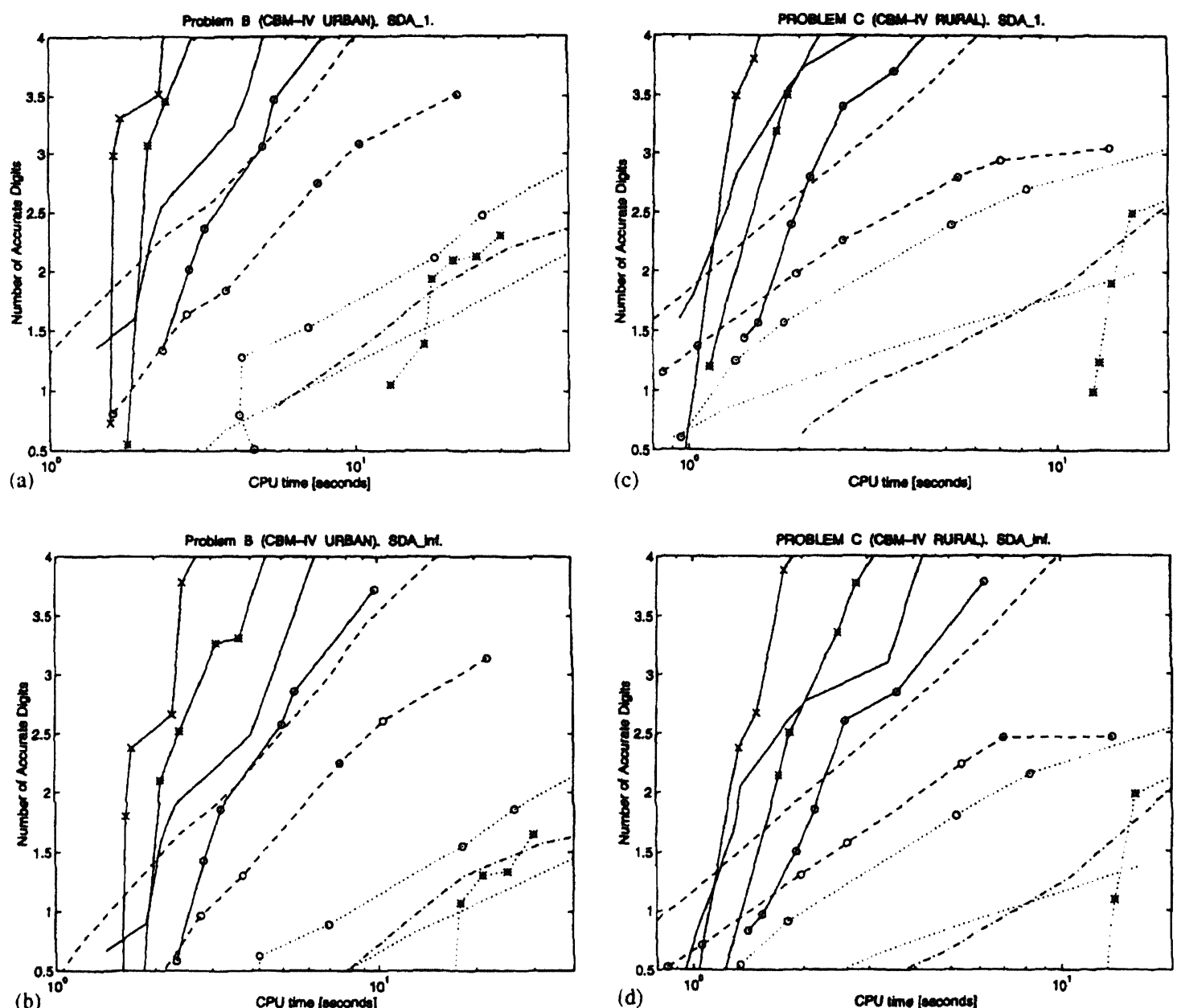

Fig. 2. Work-precision diagram for test problems $B$ and C (CBM-IV): The upper pair of diagrams correspond to problem B and the lower pair to problem C. TWOSTEP SEIDEL (dashed), TWOSTEP JACOBI (dashed with "O"), QSSA (dots), Extrapolated QSSA (dots with “O"), ET (dots with "*") CHEMEQ (dash-dots), Sparse VODE (solid), Sparse SDrRK4 (solid with "*"), Sparse RODAS (solid with " $x$ ") and LSODES (solid with "O").

scenarios are simulated with both CBM-IV and AL. They differ however in the number of reactions and species, the current problems $\mathrm{D}$ and $\mathrm{E}$ being much larger. If a standard implementation of the implicit solvers was used, their linear algebra workload would have increased as $m^{3}$, with $m$ the number of species, while for dedicated explicit integrators the workload increases linearly with $m$. Thus, at first sight, for sufficiently large problems use of explicit integrators seems to be preferable. Because we use a sparse linear algebra implementation, the situation becomes truly different. For the sparse implementation a rough estimation of the linear algebra workload is given by the number of nonzero elements in the Newton matrix. As seen in Table 1, this number increases almost linearly with $m$ for the test mechanisms considered here. This means that even for fairly large chemical systems, sparse implicit solvers may very well remain competitive. Our test results shown in Fig. 3 clearly illustrate this. For both problems all sparse implicit solvers outperform the dedicated explicit ones, with the exception of TWOSTEP SEIDEL. The gap between this code and its JACOBI version is again due to the fact that SEIDEL iterations allow the use of larger values for the minimal step size. Still, this imposed minimal step size causes convergence problems in part of the time interval, which explains the curious slopes of TWOSTEP in the range of high accuracies for the more difficult urban scenario. Noteworthy is that the onestep solver RODAS is the fastest in the $1 \%$ error region. For the more difficult urban problem, the two one-step solvers RODAS and SDRIK4 are always faster than their BDF counterparts VODE and LSODES. The explicit solver ET fails to integrate problem $D$ and is among the slowest for problem $\mathrm{E}$. The CHEMEQ diagram is in between that for QSSA and EXTRAPOLATED QSSA. The latter clearly performs better in the rural cases than in the urban ones for both CBM-IV and $\mathrm{AL}$ mechanisms.

\subsection{Problem F: the stratospheric model}

This problem has about the same dimension as the two CBM-IV problems, but the integrators perform quite differently relative to each other as shown in 

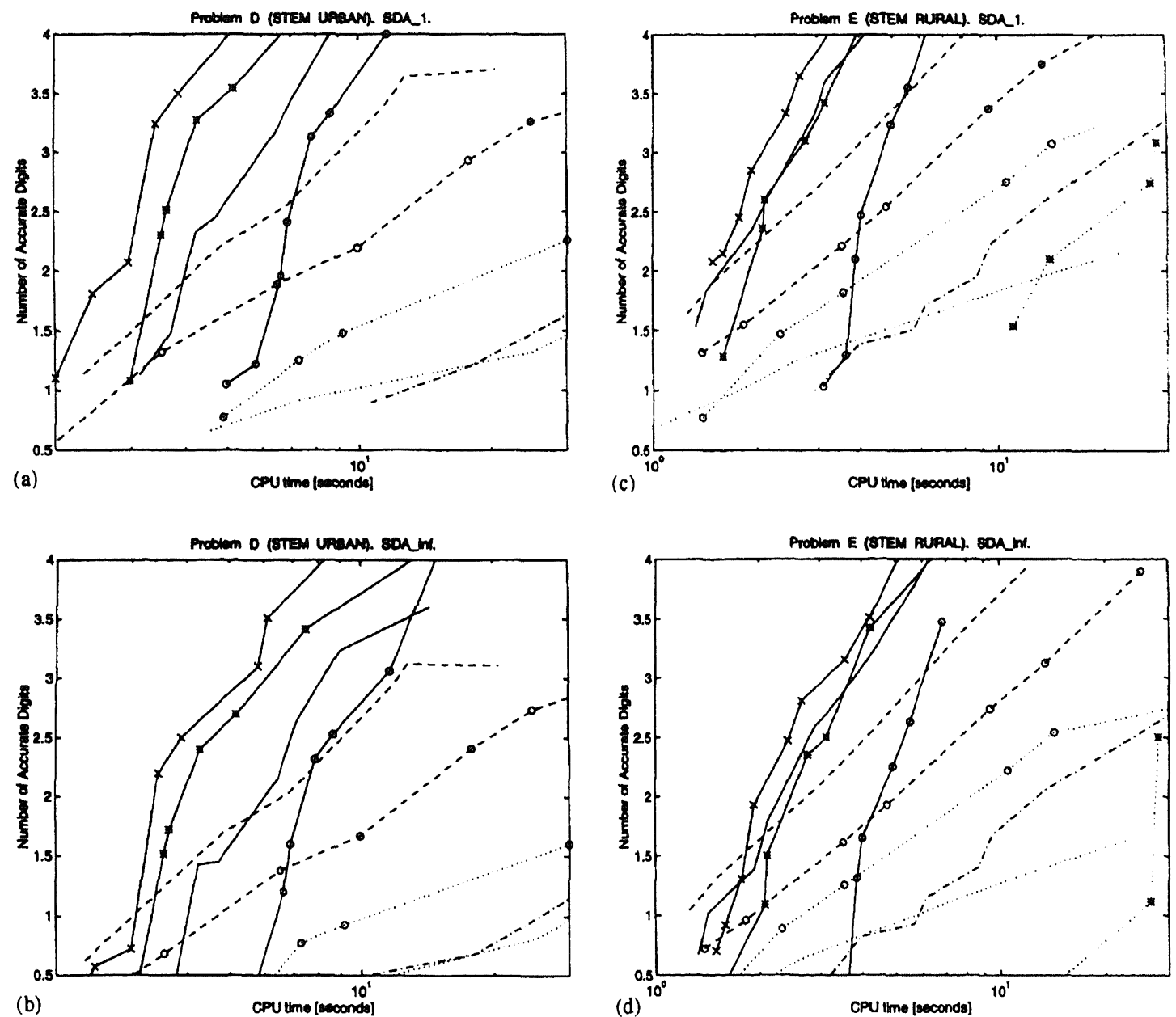

Fig. 3. Work-precision diagram for test problems $D$ and $E$ (AL): The upper pair of diagrams correspond to test problem D, while lower pair to test problem E. TWOSTEP SEIDEL (dashed), TWOSTEP JACOBI (dashed with "O"), QSSA (dots), Extrapolated QSSA (dots with "O"), ET (dots with "*"), CHEMEQ (dash-dots), Sparse VODE (solid), Sparse SDIRK4 (solid with “*”), Sparse RODAS (solid with " $\times$ ") and LSODES (solid with "O").

Fig. 4. The implicit integrators work best. There is not much difference between their performances, but there is a large gap between them and the explicit codes, with the largest for ET, CHEMEQ and the two QSSA solvers. In particular, for this problem the implicit codes require less CPU time than for CBM-IV, whereas for TWOSTEP the amount of CPU time almost remains equal. From an additional investigation we learned that the absence of emissions in the stratospheric problem must play a role here. Without emissions, concentration values vary less in between the one hour subintervals. The implicit solvers enjoy this, since as a rule they allow larger step sizes than TwoSTEP. We have checked this observation by removing the emissions in the CBM-IV model. In this case the implicit solvers also integrate much faster, the CPU timings being then similar to those obtained for the stratospheric problem.

\subsection{Problem G: the wet model}

As we have previously seen, this test problem has a large number of stiff eigenvalues, most of which cannot be associated to certain short-lived species. This makes the test problem $G$ numerically challenging in the sense that the explicit methods fail. Numerical results confirm that the explicit formulas described in Section 3 cannot solve this problem with a reasonable efficiency. We tested each routine with the loosest restrictions on step size and tolerance for which the numerical results were still meaningful. See the codes ftp.cgrer.uiowa.edu. (1996) for the exact setting of the parameters. The timings obtained for integrating the first $10 \mathrm{~s}$ (or $1 \mathrm{~h}$ ) of evolution are given in Table 10. When looking at the results, keep in mind the fact that all the implicit solvers integrate the test problem along the five days interval in less than $10 \mathrm{~s}$ CPU time. Among the dedicated integrators, TWOSTEP SEIDEL performs best. However, even this code selects very small step sizes, which is not the case when a fully implicit implementation of the BDF2 formula is used (VODE with restricted maximal order). The behaviour of the dedicated integrators is typical for standard explicit formulas applied to general stiff problems. To make the terms of comparison more 

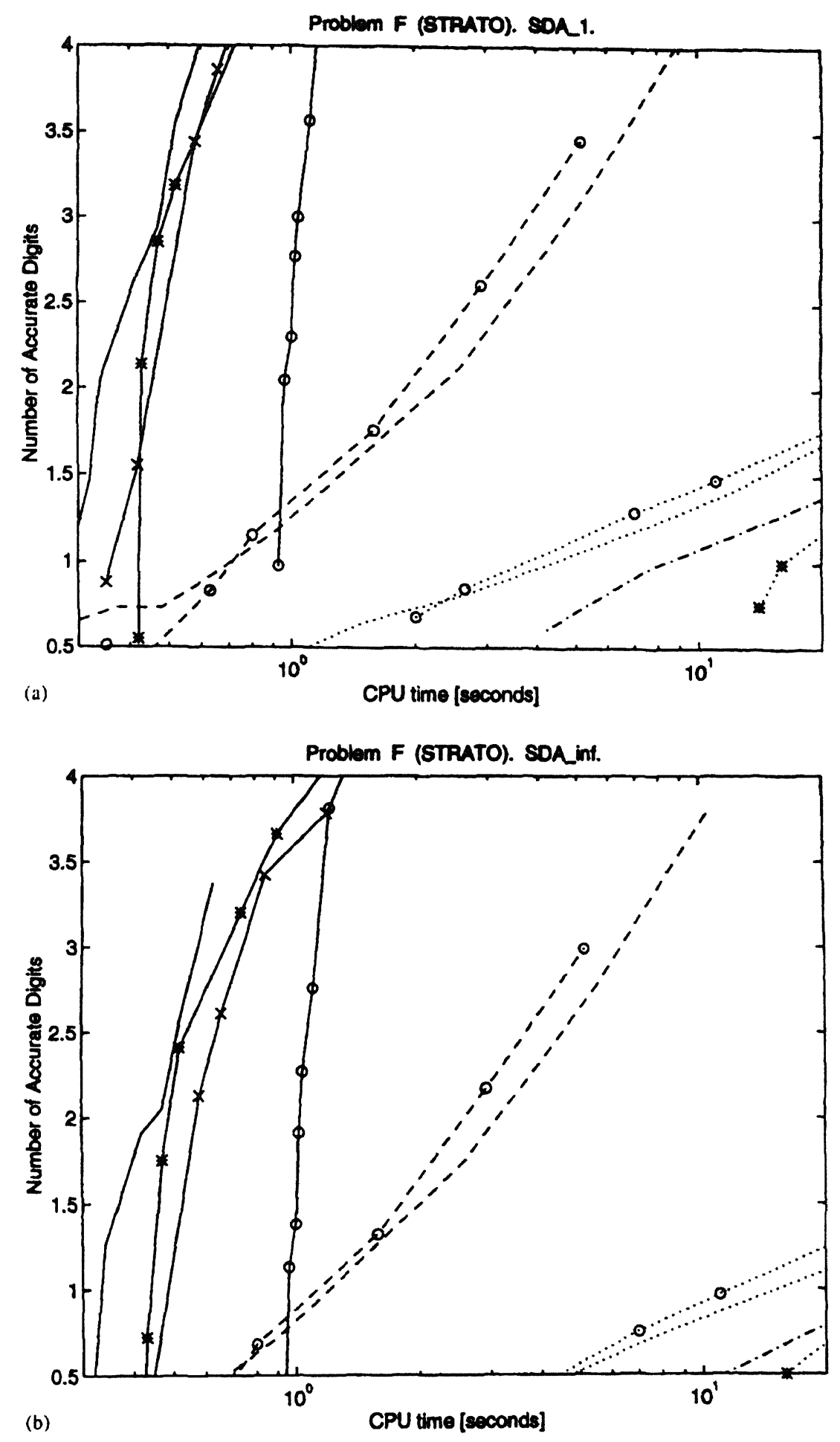

Fig. 4. Work-precision diagram for test problem F (STRATO): TWOSTEP SEIDEL (dashed), TWOSTEP JACOBI (dashed with "O"), QSSA (dots), Extrapolated QSSA (dots with "O"), ET (dots with "*"), CHEMEQ (dash-dots), Sparse VODE (solid), Sparse SDIRK4 (solid with "*"), Sparse RODAS (solid with " $x$ ") and LSODES (solid with “O”).

clear, we estimated the CPU time that would be needed to complete the five days simulation. This estimated time is given in the last column of Table 10. The explicit solver ET gives a floating point exception on this test problem and is not included in the table.
Some effort has been made to optimize the performance of the implicit solvers for this test problem. RODAS and SDIRK 4 were used with a minimal stepsize of $10^{-3} \mathrm{~s}$, while for VODE a minimum of $10^{-8} \mathrm{~s}$ appeared to be best. No minimal step size was prescribed 


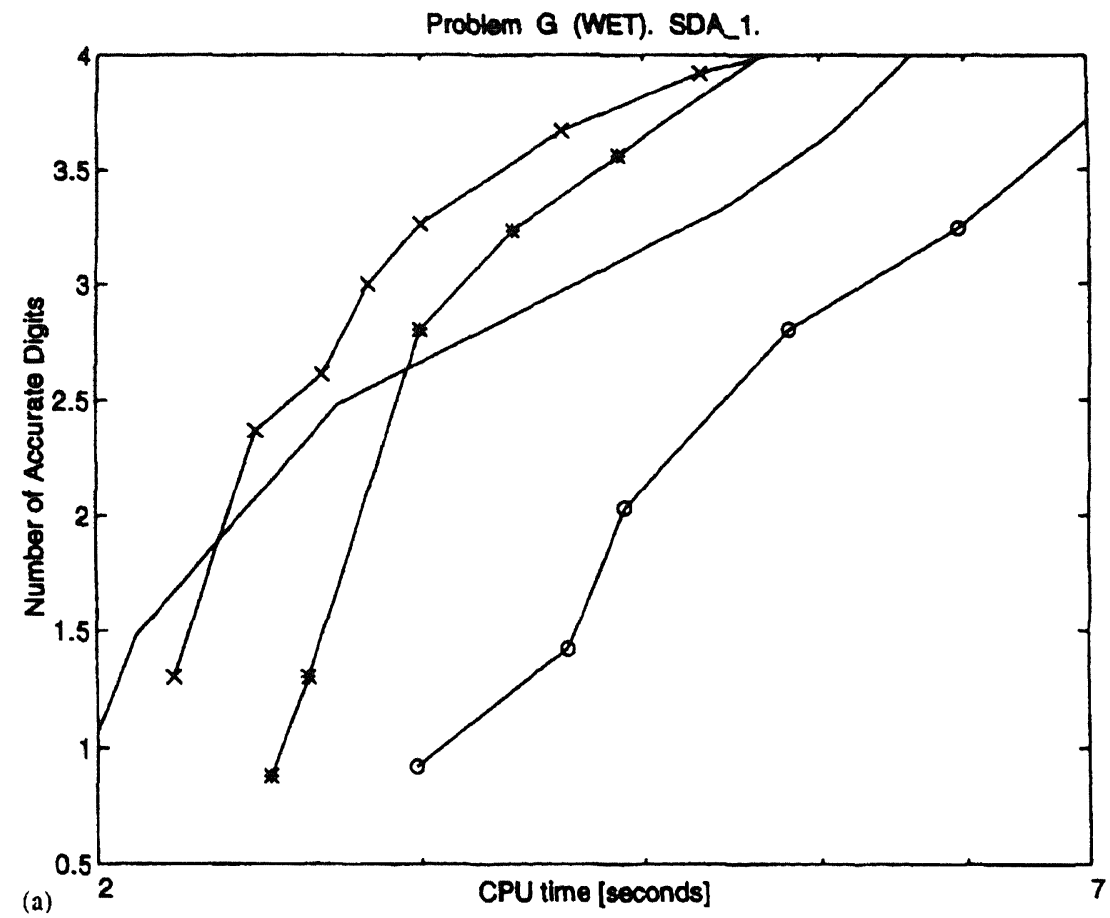

(a)

Problem G (WET). SOA inf.

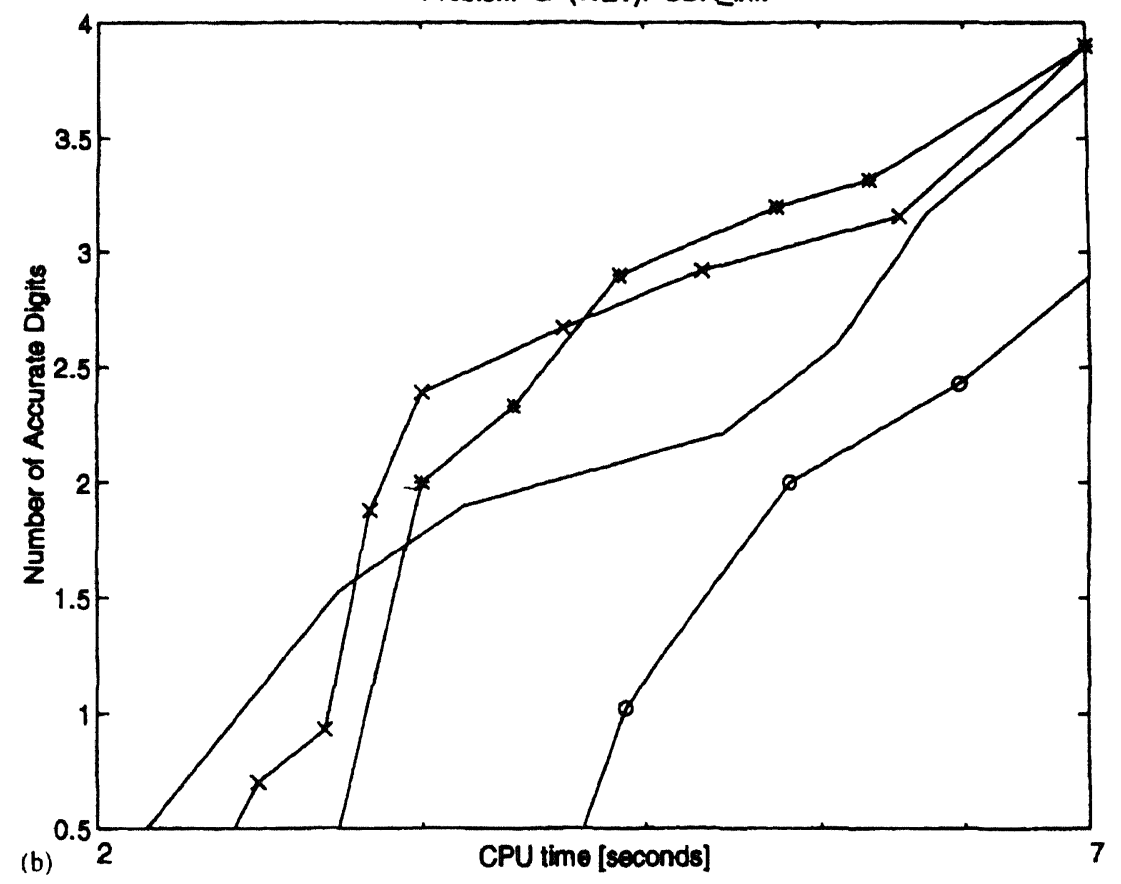

Fig. 5. Work-precision diagram for test problem G (WET): Sparse (solid), Sparse SDIRK4 (solid with "*"). Sparse RODAS (solid with " $x$ ") and LSODES (solid with "O").

for LSODES. For sparse vODE the maximal order was restricted to three. Although the original vODE had no problems integrating this model, the sparse version selected tiny step sizes when a maximal order of four or five was used. The work-precision diagram for the implicit schemes is given in Fig. 5, which shows that RODAS, VODE and SDIRK 4 perform equally well.

\section{OVERALL CONCLUSIONS AND REMARKS}

Although we have taken any reasonable precaution in implementing the models and in testing the codes, still undiscovered errors and/or less optimal settings of user parameters may have affected part of the numerical results presented here. The interested reader therefore is invited to repeat the experiments 
Table 10. The timing of dedicated integrators on test problem $\mathrm{G}$. The last column represents the estimated CPU time needed to complete the five days simulation

\begin{tabular}{lccc}
\hline Integrator & $\begin{array}{c}\text { Simulated } \\
\text { interval (s) }\end{array}$ & $\begin{array}{c}\text { CPU } \\
\text { time (min) }\end{array}$ & $\begin{array}{c}\text { Estimated } \\
\text { total CPU }\end{array}$ \\
\hline QSSA & 10 & 19 & $350 \mathrm{~d}$ \\
Extrap QSSA & 10 & 33 & $650 \mathrm{~d}$ \\
CHEMEQ & 10 & 0.5 & $15 \mathrm{~d}$ \\
TWOSTEP J & 10 & 9 & $270 \mathrm{~d}$ \\
TWOSTEP S & 3600 & 5.3 & $10.3 \mathrm{~h}$ \\
\hline
\end{tabular}

using our codes from ftp.cgrer.uiowa.edu. (1996) and to join us* in this benchmark activity. In future work we will continue evaluating hybrid and other numerical schemes. The results presented in the present paper indicate to the following conclusions and remarks:

- The sparse implicit solvers work efficiently for all problems tested here, including the gas-liquid phase one. In all the cases they give the fastest solution, when two or more accurate digits are required. In general RODAS, VODE and SDIRK4 have comparable performances, although their ranking relative to each other may differ with problem. In most test cases sparse RODAS is the fastest in the $1 \%$ error region, with VODE being the best for problems $A$ and $F$. The one-step Runge-Kutta solvers RODAS and SDIRK4 clearly work better on the urban AL problem. It should be noted that VODE appeared to be somewhat sensitive to the choice of the absolute tolerances and the choice of a minimal step size.

- Of the dedicated explicit solvers, TWOSTEP is clearly the best. This solver outperforms the other explicit solvers on all problems, often with a wide gap. TWOSTEP is advocated for gas-phase problems only. This code should not be applied to gas-liquid phase problems. In general TWOSTEP SEIDEL is more efficient than TWOSTEP JACOBI. However, Gauss-Seidel iteration must be programmed in line which makes TWOSTEP JACOBI somewhat easier to use. It is important to note that dedicated explicit solvers can sometimes be significantly improved by problemdependent modifications like lumping and/or group iteration. Of course, this requires a considerable knowledge of the reaction mechanism.

- In most cases sparse VODE is more efficient than the related sparse BDF solver LSODES. We owe this to the fact that VODE uses a dedicated sparsity technique, whereas LSODES uses the general Yale sparse matrix package. We should also mention that LSODES is used without a precribed minimal stepsize. Some additional runs with sparse VODE without a minimal step size as well, demonstrated that this plays a minor role, the difference in sparse matrix treatment being more important. Both have been applied with the extra

* Contact J.G. Verwer (janv@cwi.nl) and A. Sandu (sandu@cgrer.uiowa.edu). storage option for the Jacobian matrix so as to avoid Jacobian updates when possible.

- Since all the tested implicit solvers are general purpose ones, except for the use of sparsity, we definitely think that within this class of methods there is considerable room for efficiency improvements for atmospheric chemistry applications. The current research will therefore be continued with a search to determine also a best or near-to-best sparse implicit solver for atmospheric chemistry problems.

- The best sparse implicit solvers and the best explicit solver (TWOSTEP) should also be compared in 3D model applications. While box model tests are needed to select and develop promising ODE solvers, in real 3D transport-chemistry models other factors should be taken into account as well. Quite important is the length of the time step in the operator splitting, since this determines the number of restarts. Restarts are expensive for implicit codes and one-step methods of Runge-Kutta type have an advantage here over multistep methods. Also robustness and ease of use are important in 3D models, since a subtle tuning of the ODE code is cumbersome due to the large variety of conditions that will occur at different gridpoints. Finally, the issues of memory use, vectorization (Jacobson and Turco, 1994; Verwer et al., 1996) and parallelization are of great practical importance too. Optimal ODE solvers should be tested in a 3D software environment where vectorization and parallelization take place.

Acknowledgements-The work of the authors Sandu, Carmichael and Potra was supported in part by the DOE under grant no DE-FG02-94ER 61855 and by the Center for Global and Regional Environmental Research. The authors Verwer and Van Loon gratefully acknowledge support from the RIVM (Dutch National Institute of Public Health and Environmental Protection) for the projects EUSMOG and CIRK.

\section{REFERENCES}

Atkinson, R. D., Baulch, D. L., Cox, R. A., Hampson, R. F JR., Kerr, J. A. and Troe, J. (1989) Evaluated kinetic and photochemical data for atmospheric chemistry. J. Chem. Kinet. 21, 115-190.

Brown, P. N., Byrne, G. D. and Hindmarsh, A. C. (1989) VODE: A variable coefficient ODE Solver. SIAM J. Sci. Stat. Comput. 10, 1038-1051.

Carmichael, G. R., Peters, L. K. and Kitada, T. (1986) A second generation model for regional-scale transport/ chemistry/deposition. Atmospheric Environment 20, 173-188.

Dabdub, D. and Seinfeld, J. H. (1995) Extrapolation techniques used in the solution of stiff ODEs associated with chemical kinetics of air quality models. Atmospheric Environment 29, 403-410.

Damian-Iordache, V. and Sandu, A. (1995) KPP-A symbolic preprocessor for chemistry kinetics-User's guide. Technical Report, Department of Mathematics, University of Iowa.

Eisenstat, S. C., Gursky, M. C., Schultz, M. H. and Sherman, A. H. (1977) Yale Sparse Matrix Package.ii. The nonsymmetric codes. Research Report 114, Department of Computer Science, Yale University. 
Eisenstat, S. C., Gursky, M. C., Schultz, M. H. and Sherman, A. H. (1982) Yale Sparse Matrix Package.i. The symmetric codes. Int. J. Num. Meth. Eng. 18, 1145-1151.

Prather et. al., M. (1995) Intercomparison of tropospheric chemistry/transport models. In Scientific Assesment of Ozone Depletion, World meteorological organization.

ftp.cgrer.uiowa.edu. (1996) Ftp. site at the Center for Global and Regional Environmental Research, University of Iowa (cd pub/Ode_benchmark, mget *).

Gery, M. W., Whitten, G. Z., Killus, J. P. and Dodge, M. C. (1989) A photochemical kinetics mechanism for urban and regional scale computer modeling. J. geophy. Res. 94, 12925-12956.

Hairer. E. and Wanner, G. (1991) Solving Ordinary Differential Equations II. Stiff and Differential-Algebraic Problems. Springer, Berlin.

Hov, Ø., Isaksen, I. and Hesstvedt, E. (1978) A numerical method to predict secondary air pollutants with an application on oxidant generation in an urban atmosphere. WMO publication 510, 219-226.

Hesstvedt, E., Hov, $\oslash$. and Isaksen, I. (1978) Quasi-steadystate-approximation in air pollution modelling: comparison of two numerical schemes for oxidant prediction. Int. J. chem. Kinet. 10, 971-994.

Hindmarsh, A. C. (1983) ODEPACK: A systematized collection of ODE solvers. In R. S. Stepleman (ed.), IMACS Trans. on Scientific Computation, Vol. 1, Scientific Computing, ed. R. S. Stepleman, North Holland, Amsterdam.

Jacobson, M. Z and Turco, R. P. (1994) SMVGEAR: a sparse-matrix, vectorized Gear code for atmospheric models. Atmospheric Environment 17, 273-284.

Jay, L. O., Sandu, A., Potra, F. A. and Carmichael, G. R. (1995) Improved QSSA methods for atmospheric chemistry integration. Technical report 67, Department of Mathematics, University of Iowa.

Lurmann, F. W., Loyd, A. C. and Atkinson, R. (1986) A chemical mechanism for use in long-range transport/acid deposition computer modeling. J. geophys. Res. 91, 10,905-10,936.

McRae, G. J., Goodin, W. R. and Seinfeld, J. H. (1982) Numerical solution of the atmospheric diffusion equation for chemically reacting flows. J. comput. Phys. 45, 1-42.
Matthijssen, J. (1995) Private communication, Laboratoire d'Aerologie OMP, Toulouse, France.

Sandu, A., Potra, F. A., Damian, V. and Carmichael, G. K. (1996) Efficient implementation of fully implicit methods. for atmospheric chemistry. J. Comp. Phys. 129, 101110.

Saylor, R. D and Ford, G. D. (1995) On the comparison of numerical methods for the integration of kinetic equations in atmospheric chemistry and transport models. Atmospheric Environment 29, 2585-2593.

Simpson, D., Andersson-Sköld, Y. and Jenkin, M. E. (1993) Updating the chemical scheme for the EMEP MSC-W oxidant model: current status. Technical Report 2/93. EMEP MSC-W. The Norwegian Meteorological Institute, Oslo.

van Loon, M. (1995a) Numerical smog prediction I: The physical and chemical model. CWI Report NM-R9411, Center for Mathematics and Computer Science, Amsterdam.

van Loon, M. (1995b) Numerical smog prediction Il: Grid refinement and its application to the Dutch smog prediction model. CWI Report NM-R9523, Center for Mathematics and Computer Science, Amsterdam.

Verwer, J. G. (1994) Gauss-Seidel iterations for stiff ODEs from chemical kinetics. SIAM J. Sci. Comput. 15. $1243-1250$

Verwer, J. G., Blom, J. G., van Loon, M. and Spee, E. J. (1996) A comparison of stiff ODE solvers for atmospheric chemistry problems. Atmospheric Environment 30, 49-58.

Verwer, J. G. and Simpson, D. (1995) Explicit Methods for stiff ODEs from atmospheric chemistry. Appl. Numer. Math. 18, 413-430.

Verwer, J. G., Blom, J. G. and Hundsdorfer, W. H. (1996) An implicit-explicit approach for atmospheric transportchemistry problems. Appl. Numer. Math. 20, 191.209.

Yamartino, R., Scire, J., Carmichael, G. R. and Chang, Y. S. (1992) The CALGRID mesoscale photochemical grid model. Atmospheric Environment 26A, 1493-1512.

Young, T. R. and Boris, J. P. (1977) A numerical technique for solving stiff ODEs associated with the chemical kinetics of reactive flow problems. J. phys. Chem. 81, 2424-2427. 